\title{
Primerjava obeh prepisov Beethovnove Seste simfonije
}

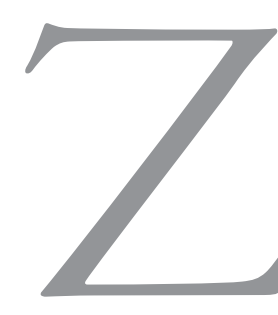

aznamki in opombe tega revizijskega poročila se nanašajo na razlike med partiturama prvega avtoriziranega prepisa Beethovnove $\check{S}$ este simfonije, »Pastoralne«, ki ga hrani Beethovnova hiša v Bonnu (Beethoven-Haus Bonn, NE I46), ${ }^{\text {in }}$ in drugega prepisa, ki ga hrani Narodna in univerzitetna knjižnica (NUK) v Ljubljani (NUK, Glasbena zbirka, M tre Be-

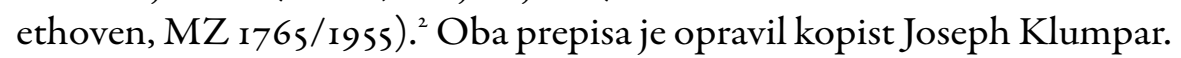
$\mathrm{V}$ obeh so prisotni Beethovnovi posegi, $\mathrm{v}$ prvem prepisu številni zapisani z njegovim značilnim rdečkastim svinčnikom, $\mathrm{v}$ drugem pa so skoraj vsi posegi upoštevani. V drugem prepisu $\mathrm{z}$ eno samo izjemo takta $54 \mathrm{v}$ drugem stavku rdečkastega svinčnika ni zaslediti. Vse njegove pripombe so zapisane z navadnim svinčnikom, kar daje slutiti, da so bile napisane med vajami za izvedbo decembra $18 \circ 8$. Tako lahko pojmujemo drugi, »Ljubljanski « prepis simfonije kot čistopis.

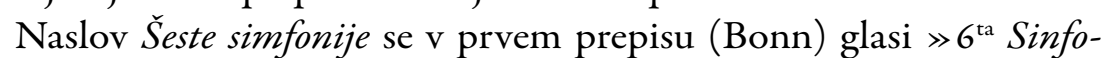
nia pastorella «. Nad njo je pripis $\gg$ Pastorale «. V drugem prepisu (Ljubljana) je naslov » Beethovens Symphonie pastorale $\mathrm{N}^{\circ} 6 . \ll$.

I Dostopno v: Beethoven-Haus Bonn, https://www.beethoven.de/de/digitales-archiv.

2 Dostopno v: Beethoven, Ludwig van (1808). Sinfonia pastorale. URN:NBN:SI:DOC-98LTR6Yofrom http://www.dlib.si.
Načeloma so oznake za cresc. pisane brez pike v okrajšavi »cres:«, prav tako pizz., vendar v obliki »pizzi«. Skoraj vedno so Beethovnovi posegi zapisani z rdečim svinčnikom, kasneje pa jih je kopist prevlekel $s$ črnilom. To v revizijskem poročilu posebej ni več omenjeno.

Kratice in oznake so enake kot $v$ Revizijskem poročilu. 
I. stavek

\begin{tabular}{|c|c|c|c|c|}
\hline Takt & Instrument & $\begin{array}{l}\text { Prvi avtorizirani prepis (Bonn) } \\
\text { Beethovnov poseg }\end{array}$ & Instrument & $\begin{array}{c}\text { Drugi prepis }-\gg \text { čistopis } \ll(\text { Ljubljana }) \\
\text { Beethovnov poseg }\end{array}$ \\
\hline 94,95 & Vni I, vla & Razvezaj pred drugo noto. & & Razvezaji samo v vla. \\
\hline 97 & Tutti & & & Upoštevano. \\
\hline 197 & Cor, fg, Basi & $\begin{array}{l}\text { Oznaka »cresc. poco a poco《, } \\
\text { ki jo je kopist kasneje prevlekel s črnilom. }\end{array}$ & & Upoštevano. \\
\hline $206-208$ & $\begin{array}{l}\text { Vni II., cor, } \\
\text { fg }\end{array}$ & $\begin{array}{l}\text { Kopist je kljub ligaturi v vsakem taktu notiral } \\
\text { višaj, Beethoven ga je črtal. }\end{array}$ & & Upoštevano. \\
\hline 213 & Vla & Tretja nota razvezaj. & & Upoštevano. \\
\hline 218 & Vni I., 2. fl & Prečrtan vnovičji višaj (kljub ligaturi). & & Upoštevano. \\
\hline 229 & Vni II. & Dopisana oznaka »diminuendo«. & & Upoštevano. \\
\hline 234 & Vni I., fg & Drugi noti dopisan razvezaj. & & $\begin{array}{l}\text { Ni upoštevano. Verjetno je kopist menil, } \\
\text { da se igra per analogiam. }\end{array}$ \\
\hline 243 & $\mathrm{Fl}$ & $\begin{array}{l}\text { Lok preko dveh taktov in v naslednjem taktu } \\
\text { še preko dveh not. }\end{array}$ & & Upoštevano. \\
\hline 284 & & & Vni I. & Beethovnov poseg: trilerju dodan dvojni založek. ${ }^{3}$ \\
\hline 289 & & & Vni I. & Prva nota v trioli oddvojena. \\
\hline 301 & Vni I. & Trije takti pavze. ${ }^{4}$ & & $\begin{array}{l}\text { Dodana opomba } \gg \text { simile } \ll \\
\text { (3 takti od 2. takta naprej). }\end{array}$ \\
\hline 302 & Vni II. & Pred četrto noto dodan nižaj $(b)$. & & Upoštevano. \\
\hline 344 & Vni II. & Dodana opomba »arco«. & & Upoštevano. \\
\hline
\end{tabular}

3 Zelo verjetno je kopist Klumpar ta dodatek zapisal po originalu - zapis je napisan s črnilom.

4 V avtografu je od drugega takta naprej vpisan znak $\gg$ simile $\ll$ ! 


\begin{tabular}{|c|c|c|c|c|}
\hline Takt & Instrument & $\begin{array}{l}\text { Prvi avtorizirani prepis (Bonn) } \\
\text { Beethovnov poseg }\end{array}$ & Instrument & $\begin{array}{c}\text { Drugi prepis }-\gg \text { čistopis } \ll(\text { Ljubljana }) \\
\text { Beethovnov poseg }\end{array}$ \\
\hline 345 & Vlc & Dodana opomba $\gg$ arco $\ll$ & & Upoštevano. \\
\hline 358 & $\mathrm{Cb}$ & $\begin{array}{l}\text { Dodana opomba } \gg \text { arco } \ll \text { in } \gg c r e s c \ll . \\
\text { Zapis samo na črtovju cb, kot da vlc tacet. }\end{array}$ & & $\begin{array}{l}\text { Nejasno mesto. Opomba ni upoštevana, } \\
\text { kot da vlc nadaljuje svojo linijo. } \\
\text { Zapis samo na črtovju cb! }\end{array}$ \\
\hline 390 & Vni II. & Dodana opomba $\gg$ arco $\ll$. & & Upoštevano. \\
\hline 414 & & & Vni I. & Oznaki $p$ dodan še dodaten $p$, torej $p p$. \\
\hline \multicolumn{5}{|c|}{ 2. stavek } \\
\hline Takt & Instrument & $\begin{array}{l}\text { Prvi avtorizirani prepis (Bonn) } \\
\text { Beethovnov poseg }\end{array}$ & Instrument & $\begin{array}{c}\text { Drugi prepis }-\gg \text { čistopis } \ll(\text { Ljubljana }) \\
\text { Beethovnov poseg }\end{array}$ \\
\hline Začetek & Vlc & & & $\begin{array}{l}\text { Beethovnov poseg v 2. prepisu: pred partom Vlc } \\
\text { je s svinčnikom dopisano »Due (Violoncelli) } \\
\text { primo e secondo solo« ter pod njo »Con Sordino«, } \\
\text { na spodnjem robu strani pa je prav tako } \\
\text { z Beethovnovo roko dopisano »gli Violoncelli } \\
\text { tutti coi Bassi《. Zaradi obreze v živi rob je tekst } \\
\text { zelo težko čitljiv. }\end{array}$ \\
\hline 27 & Vlc & $\begin{array}{l}\text { Pred tretjo noto druge triole prečrtan odvečen } \\
\text { predznak; enako v naslednjem taktu. }\end{array}$ & & Ni upoštevano \\
\hline 40 & Vla & $\begin{array}{l}\text { Na drugo dobo pred drugo noto prečrtan } \\
\text { odvečen predznak. }\end{array}$ & & Ni upoštevano. \\
\hline & Vlc & $\begin{array}{l}\text { Na četrto dobo pred zadnjo noto triole dodan } \\
\text { nižaj. }\end{array}$ & & Upoštevano. \\
\hline & $\mathrm{Cb}$ & Dodana oznaka »pizz.«. & & Upoštevano. \\
\hline
\end{tabular}




\begin{tabular}{|c|c|c|c|c|}
\hline Takt & Instrument & $\begin{array}{c}\text { Prvi avtorizirani prepis (Bonn) } \\
\text { Beethovnov poseg }\end{array}$ & Instrument & $\begin{array}{c}\text { Drugi prepis - »čistopis « (Ljubljana) } \\
\text { Beethovnov poseg }\end{array}$ \\
\hline 44 & Cor & $\begin{array}{l}\text { Dodani še ena celinka ter dva loka za povezavo } \\
\text { v naslednji takt. }\end{array}$ & & $\begin{array}{l}\text { Notirani sta dve celinki, loka pa nista } \\
\text { upoštevana. }\end{array}$ \\
\hline \multirow[t]{2}{*}{47} & Vla, vlc, cb & Dodana oznaka »cresc.«. & & $\begin{array}{l}\text { Upoštevano, razen pri cb, kjer je cresc. dopisan } \\
\text { dva takta kasneje. }\end{array}$ \\
\hline & & & Vni I. & Na tretjo dobo dodana oznaka »cresc.«. \\
\hline \multirow[t]{2}{*}{48} & $\mathrm{Cl}$, cor & Dodana oznaka $\gg$ cresc. $\ll$. & & Upoštevano. \\
\hline & Vni II. & Dodana oznaka $\gg$ arco«. & & Upoštevano. \\
\hline \multirow[t]{2}{*}{49} & Vni II., Vla & Dodana oznaka $\gg$ cresc. $\ll$. & & \\
\hline & Tutti & $\begin{array}{l}\text { Na tretjo dobo dodan } s f \text { ter na četrto dobo } \\
\text { diminuendo. }\end{array}$ & & Upoštevano. \\
\hline 54 & $\begin{array}{l}\text { Vlc soli } \\
\text { Vni I. }\end{array}$ & Dodana oznaka $\gg p i z z . \ll$. & & $\begin{array}{l}\text { Upoštevano. } \\
\text { Beethovnova korektura: dodane pike } \\
\text { nad šestnajstinkami I. dobe spremenjene v kline. }\end{array}$ \\
\hline 58 & Vlc soli & Dodana oznaka $\gg$ arco $\ll$. & & Upoštevano. \\
\hline 6I & Vlc soli & $\begin{array}{l}\text { Na tretjo in četrto dobo črtana odvečna višaj }(f i s) \\
\text { in razvezaj }(e) \text {. }\end{array}$ & & Upoštevano. \\
\hline \multirow[t]{2}{*}{68} & $\mathrm{Ob}$ & Na zadnjo osminko dodan nižaj. & & Upoštevano. \\
\hline & $\mathrm{Cl}$ & $\begin{array}{l}\text { Na četrto dobo obema instrumentoma } \\
\text { dodan nižaj. }\end{array}$ & & Upoštevano. \\
\hline 76 & Tutti & Dodana oznaka $\gg p \ll$. & & Upoštevano. \\
\hline 78 & $\mathrm{Cl}$ & Dodana vezaja v naslednji takt. & & Upoštevano. \\
\hline 82 & Vni II., Vla & Dodana oznaka $\gg$ simile $\ll \mathrm{v}$ drugem delu takta. & & Upoštevano in izpisano. \\
\hline
\end{tabular}




\begin{tabular}{|c|c|c|c|c|}
\hline Takt & Instrument & $\begin{array}{l}\text { Prvi avtorizirani prepis (Bonn) } \\
\text { Beethovnov poseg }\end{array}$ & Instrument & $\begin{array}{c}\text { Drugi prepis }-\gg \text { čistopis } \ll(\text { Ljubljana }) \\
\text { Beethovnov poseg }\end{array}$ \\
\hline 84 & $\mathrm{Cb}$ & $\begin{array}{l}\text { Dodani oznaki } \gg \text { arco } \ll \text { in } \gg \text { cresc. } \\
\text { ter pred prvo noto dodan razvezaj. }\end{array}$ & & Upoštevano. \\
\hline 86 & $\begin{array}{l}\text { Legni, Vlc } \\
\text { soli }\end{array}$ & Dodan $\gg$ diminuendo« preko dveh taktov. & & Upoštevano. \\
\hline 87 & $\mathrm{Cb}$ & Dodana oznaka $\gg p i z z . \ll$. & & \\
\hline 9I & & & $\mathrm{Fl}$ & $\begin{array}{l}\text { Beethovnov pripis: V spodnji vrstici je } \\
\text { s svinčnikom dopisana linija fl v spodnji oktavi. }\end{array}$ \\
\hline 109 & Vlc soli & Na zadnjo dobo dodana vezava. & & $\begin{array}{l}\text { Upoštevano, v naslednjem taktu pa vezavi } \\
\text { manjkata. }\end{array}$ \\
\hline IIO & $\mathrm{Cb}$ & Dodana oznaka »arco«. & & Upoštevano. \\
\hline II9 & Vni I. & Dopisana oznaka »cresc.«. & & Upoštevano. \\
\hline \multirow[t]{2}{*}{120} & Vni II. & Dopisana oznaka $\gg$ arco $\ll$ in $\gg c r e s c . \ll$. & & Upoštevano. \\
\hline & Cor & Dopisana oznaka »cresc.«. & & Upoštevano. \\
\hline I2I & Tutti & $\begin{array}{l}\text { Na tretjo dobo dopisan } s f \text {, na četrto dobo pa znak } \\
\text { za diminuendo. }\end{array}$ & & Upoštevano. \\
\hline $\begin{array}{l}122 \\
136\end{array}$ & Tutti & Na prvo dobo oznaka $\gg p \ll$ & $\mathrm{Fl}$ & $\begin{array}{l}\text { Upoštevano. } \\
\text { Beethovnov pripis: s svinčnikom dopisana vezava } \\
\text { obeh not na začetku takta. }\end{array}$ \\
\hline 138 & Tutti & Dopisana oznaka »cresc.« in na tretjo dobo $s f$. & & Upoštevano. \\
\hline 139 & Tutti & Dodana oznaka $\gg p \ll$ & & Upoštevano. \\
\hline
\end{tabular}


3. stavek

\begin{tabular}{|c|c|c|c|}
\hline Takt & Instrument & $\begin{array}{l}\text { Prvi avtorizirani prepis (Bonn) } \\
\text { Beethovnov poseg }\end{array}$ & $\begin{array}{c}\text { Drugi prepis }-\gg \text { čistopis } \ll(\text { Ljubljana }) \\
\text { Beethovnov poseg }\end{array}$ \\
\hline IO & Vni I., fl & Premaknjen predznak iz tretje na drugo noto. & Predznaka v tem taktu ni. \\
\hline 17 & Vni I., vla & Razvezaj na prvo noto. & Ni oznake. \\
\hline 33 & Vni I. in II. & Dodan razvezaj na zadnjo dobo. & Upoštevano. \\
\hline 39 & $\mathrm{Cb}$ & Nerazumljiva pripomba. & \\
\hline 95 & $\mathrm{Fg}$ & Dodana oznaka $\gg$ fagotto $2^{d o} \ll$ & Upoštevano. \\
\hline II 4 & $\mathrm{Cl}$ & Dodan vezaj čez vse tri četrtinke. & Upoštevano. \\
\hline 190 & Basi & Na drugo dobo dodan razvezaj prvi noti. & Ni oznake. \\
\hline 197 & Basi & Predzadnji noti v taktu dodan razvezaj. & Upoštevano. \\
\hline 198 & Basi & Pred prvo noto na drugi dobi dodan razvezaj. & Upoštevano. \\
\hline 221 & $\begin{array}{l}\text { Vni I. in II., } \\
\text { vla }\end{array}$ & Na prvo dobo dodan razvezaj. & Ni oznake. \\
\hline 238 & $\mathrm{Ob}, \mathrm{fg}$ & Dodani znaki za staccato (klini). & Upoštevano. \\
\hline $24 \mathrm{I}$ & $\mathrm{Cl}$ & Idem. & \\
\hline 307 & $\mathrm{Fg}$ & Znova pripomba $\gg$ fagotto $2^{d o} \ll$. & Upoštevano. \\
\hline 394 & Vla, basi & Znova na drugo dobo razvezaj. & Ni oznake. \\
\hline 397 & Vla & Razvezaj pred zadnjo noto. & Upoštevano. \\
\hline 404 & Basi & Pred drugo šestnajstinko na prvi dobi višaj. ${ }^{6}$ & Ni upoštevano. \\
\hline
\end{tabular}




\begin{tabular}{|c|c|c|c|}
\hline Takt & Instrument & $\begin{array}{l}\text { Prvi avtorizirani prepis (Bonn) } \\
\text { Beethovnov poseg }\end{array}$ & $\begin{array}{cc}\text { Instrument } & \text { Drugi prepis }-» \text { čistopis } \ll(\text { Ljubljana }) \\
\text { Beethovnov poseg }\end{array}$ \\
\hline 3 & Vni I. & Pike nad prvimi tremi notami. & Ni označeno. \\
\hline $2 \mathrm{I}$ & Basi & $\begin{array}{l}\text { Razdelitev na violončele (kvintuole) } \\
\text { in base (kvartuole). }\end{array}$ & Upoštevano. \\
\hline 58 & Vni I. & Pred tretjo noto nižaj (kot opomnik). & Upoštevano. \\
\hline 106 & 2. Pos & Nota $c$ popravljena na $e s$ in vezana čez takt. ${ }^{7}$ & $\begin{array}{l}\text { V tem taktu sta notirana altovski } \\
\text { in tenorski ključ. }\end{array}$ \\
\hline II2 & Vni I. & Znova notiran nižaj pred noto. & Upoštevano. \\
\hline
\end{tabular}

\section{5. stavek}

\begin{tabular}{|c|c|c|c|}
\hline Takt & Instrument & $\begin{array}{l}\text { Prvi avtorizirani prepis (Bonn) } \\
\text { Beethovnov poseg }\end{array}$ & $\begin{array}{c}\text { Instrument } \quad \begin{array}{c}\text { Drugi prepis }-\gg \text { čistopis } \ll(\text { Ljubljana }) \\
\text { Beethovnov poseg }\end{array} \\
\end{array}$ \\
\hline 5 & Cor & Dodana oznaka $\gg$ Solo «. & Upoštevano. \\
\hline 80 & $\mathrm{Cl}, \mathrm{fg}$ & $\begin{array}{l}\text { Od tretje note naprej zahtevana vezava } \\
\text { še čez cel naslednji takt. }\end{array}$ & Upoštevano. \\
\hline 83 & $\mathrm{Cl}, \mathrm{fg}$ & Posebej so notirani vsi predznaki. ${ }^{9}$ & Upoštevana sta prvi in tretji predznak, drugi ne. \\
\hline 85,90 & I. $\mathrm{cl}$ & Pred tretjo noto razvezaj. & Upoštevano. \\
\hline $87,9 \mathrm{I}$ & 2. $\mathrm{cl}$ & Pred prvo noto razvezaj. & Upoštevano. \\
\hline 89 & I. $\mathrm{cl}$ & Idem. & Upoštevano. \\
\hline
\end{tabular}

7 Verjetno je kopist pojmoval altovski trombon kot notiran v altovskem ključu in tenorski kot notiran v tenorskem ključu, tako kot je to eksplicitno storil v drugem

8 Če je altovski ključ mišljen za altovski trombon in tenorski za tenorski trombon, ostaja interval enak.

9 Predložek, pred drugo noto in pred zadnjo, glede na to, da se nahajajo v različnih instrumentih. 


\begin{tabular}{|c|c|c|c|c|}
\hline 292 & Takt & Instrument & $\begin{array}{l}\text { Prvi avtorizirani prepis (Bonn) } \\
\text { Beethovnov poseg }\end{array}$ & $\begin{array}{c}\text { Drugi prepis }->\text { čistopis } \ll(\text { Ljubljana }) \\
\text { Beethovnov poseg }\end{array}$ \\
\hline \multirow{14}{*}{ 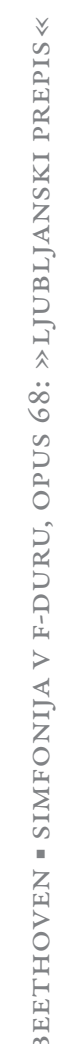 } & 93 & I. $\mathrm{cl}$ & Pred prvo noto višaj. & Upoštevano. \\
\hline & 97 & Vni II. & Pred prvo noto nižaj. & Upoštevano. \\
\hline & 99 & Vni II. & Na drugo dobo pred tretjo šestnajstinko razvezaj. & Upoštevano. \\
\hline & 100 & Vlc & Obe prvi noti povezani. & Upoštevano. \\
\hline & IOI & 2. $\mathrm{cl}$ & Pred prvo noto višaj. & Upoštevano. \\
\hline & & Basi & $\begin{array}{l}\text { Zadnja nota v taktu opremljena s kajlo } \\
\text { (enako takt kasneje). }\end{array}$ & Kajla označena šele takt kasneje. \\
\hline & III & Vni I. & Obe skupini šestnajstink pod lokom. & Enako. \\
\hline & 120 & Vni I. & Dodan nižaj na prvo dobo (kot opomnik). & Brez nižaja. \\
\hline & 178 & $\mathrm{Fg}$ & Dodana oznaka $\gg p \ll$ & Upoštevano. \\
\hline & 2 II & Vla & Pred predzadnjo šestnajstinko dopisan višaj. & Upoštevano. \\
\hline & 213 & $\mathrm{Ob}$ & Od te note vezaj čez cel drugi takt. & Upoštevano. \\
\hline & 223 & 2. $\mathrm{Ob}$ & Razvezaj pred noto. & Razvezaj manjka. \\
\hline & 231 & $\mathrm{Fl}, \mathrm{ob}$ & Dopisan lok čez tri takte. & Upoštevano. \\
\hline & 248 & Basi & Tudi tu dopisan $\gg$ sotto voce $\ll$. & Oznaka manjka. \\
\hline
\end{tabular}

